\title{
A CLOUD-BASED PLATFORM FOR IFC FILE ENRICHMENT WITH SECOND-LEVEL SPACE BOUNDARY TOPOLOGY
}

\author{
Georgios N. Lilis ${ }^{1}$, Kyriakos Katsigarakis ${ }^{2}$, Georgios I. Giannakis ${ }^{3}$, and Dimitrios Rovas ${ }^{4}$
}

\begin{abstract}
To facilitate the automatic generation of building energy performance simulation models from BIM data sources and support multiple building designs almost simultaneously in a district environment, a cloud-based platform which uses containerized micro-services for the generation of the required building's secondlevel space boundary topology from IFC geometric data, is introduced. The platform uses as input, in a parallel fashion, multiple IFC files, and executes the Common Boundary Intersection Projection (CBIP) algorithm, for the generation of the enhanced IFC files containing the second-level space boundaries of all buildings. The platform utilizes auto-scaling feature that automatically adds and removes CBIP instances depending upon actual usage. In order to test the functionalities of the platform, certain IFC data files have been uploaded to its repository.
\end{abstract}

Keywords: IFC, BIM, Second-level Space Boundaries, Building Energy Performance simulation, District.

\section{INTRODUCTION}

Build Energy Performance (BEP) simulations is a valuable tool for design and performance assessment of a building. Setting up the simulation model involves a labourintensive (and non-value producing) stage where the geometry has to be prescribed. The geometric configuration requires prescribing the topology where thermal interactions are taking places, as well as product information (building architectural elements) that includes walls, windows and internal partitions. For new, but also for existing buildings, this geometric information might be available in Building Information Models (BIM) created using BIM authoring tools, that nowadays are gaining acceptance and popularity.

Multiple attempts establishing links between BIM and BEP tools have been attempted which include: the IDF Generator (Bazjanac V. , 2009), developed at the Lawrence Berkeley National Laboratory (LBNL), works in conjunction with the Geometry Simplification Tool (GST) and transforms IFC-format building geometry into EnergyPlus input-data file (IDF) and the RIUSKA (Jokela, 1997), developed by Granlund, uses the DOE-2.1 (Hirsch, 2014) thermal simulation engine and imports the building geometry from an IFC file, utilizing the BSPro server middleware (Karola, 2002).

1 Postdoctoral Researcher, Department of Production engineering and Management, Technical University of Crete, Chania, Greece, georgios.lilis@gmail.com.

2 Researcher, Department of Production engineering and Management, Technical University of Crete, Chania, Greece, katsigarakis@gmail.com.

3 Postdoctoral Researcher, Department of Production engineering and Management, Technical University of Crete, Chania, Greece, georgios.giannakis@gmail.com.

4 Lecturer, Bartlett School Env. Energy \& Resources, University College London, London, UK, d.rovas@ucl.ac.uk. 
However, the geometric representations of the architectural elements, contained in BIM files, cannot be used directly as inputs to building energy performance simulation programs as the building second-level space boundary surfaces, i.e. the surfaces through which thermal energy flows, either among internal building spaces or between a building space and the buildings' outside environment (air/ground), are missing. Consequently, in order to satisfy such information and facilitate the information transfer between BIM models and BEP simulation tools (O'Donnell, 2014; Bazjanac V. , 2008), a semi-automatic way of generating the building's second-level space boundary surface topology from its geometric data exported from a BIM authoring program appears to be of paramount importance.

Such transformation process can be implemented using Common Boundary Intersection Projection (CBIP) algorithm (Lilis G. N., 2017) provided that certain data requirements are satisfied and the geometric data contained in the respective BIM files are free from design errors. The operations which include the main transformation process and the data completeness and correctness checks, are implemented on Industry Foundation Class BIM files, in a cloud service framework as illustrated in the outline of Figure 1 and is the main topic of the present work.

The introduced platform uses distributed resources, in order to accommodate multiple building designs from a district environment and performs a two-stage process, displayed in Figure 1 for every building, in a parallel fashion. Initially, an error checking operation (stage 1), is implemented on every input IFC file, before being inserted into the main CBIP algorithm (stage 2). The main output of the platform is an enriched IFC file, containing the second-level space boundaries. If certain error types are detected certain error messages are communicated back to the user.

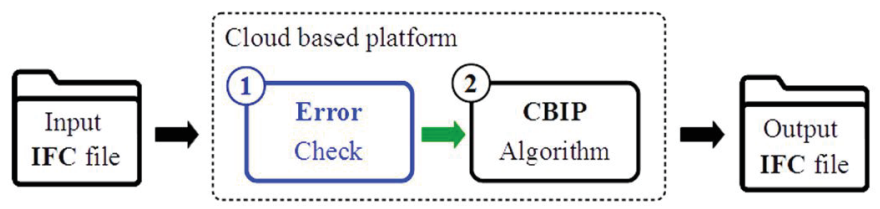

Figure 1: Outline of the proposed cloud based platform.

\section{DATA QUALITY}

The data contained in the input IFC files should conform to specific quality rules, which include completeness and correctness criteria, before being processed by the main CBIP algorithm. The completeness rules, discussed in section 2.1, ensure that the available IFC data are suitable for the second level space boundary topology generation, while the correctness rules, described in sections 2.2 , guarantee the accuracy of this topology which will be used in order to enrich the final IFC output file.

\subsection{Completeness criteria}

Input IFC files should conform to specific data completeness rules, in order for CBIP algorithm to generate the second-level space boundaries of the buildings and enrich the input IFC files. These rules are:

- Ground boundary condition. The input IFC file should contain the IfcSite class which in turns should have a Solid Model as its IfcShapeRepresentation. In other words the site of the building should be defined in three dimensions by a solid 
geometrical model. This requirement is important in order to define the ground boundary conditions of the building in the boundary surface topology.

- Conditioned spaces. Every building should contain at least one internal building space volume defined by the IfcSpace class which as in the case of IfcSite, should have a SolidModel as its IfcShapeRepresentation. This requirement guarantees that, second-level space boundary surfaces, related to the internal building spaces, will be generated by CBIP algorithm in stage 2 .

\subsection{Correctness criteria}

Three error types hinder the accurate second-level space boundary topology generation by CBIP algorithm (Lilis G. N., 2015). These error types are: clashes, space definition errors and surface orientation errors. If errors in the input IFC files are detected, certain messages are communicated back to the user depending on the error type.

\section{BOUNDARY SURFACE TOPOLOGY GENERATION}

The input IFC files, without being necessary error free (passed stage 1, Figure 1), can be used as input to the main second-level space boundary topology generation algorithm (CBIP) (stage 2, Figure 1). A detailed description of this algorithm can be found in (Lilis G. N., 2017). In short, as its name reveals, the Common Boundary Intersection Projection (CBIP) algorithm has two main stages. In the first, the common boundary stage, the common boundaries (CBs) shared by attached boundary representations of architectural element pairs (wall-slab, wall-space, ...), are identified and collected. In the second, the intersection projection stage, the CBs defined in the first stage are projected to each other, using proximity and surface orientation criteria, in order to generate the elements of the boundary surface topology of the building, demonstrated in Figure 2, which include:

1. Thermal elements. These are opaque elements of the boundary surface topology which impede thermal flow and store temporally amounts of heat (have thermal mas.

2. Shading elements. These boundary surface topology elements block direct sunlight.

3. Opening elements. These elements depending on their state block or allow thermal flow without storing heat.

4. Air boundary elements. These are imaginary boundaries used to subdivide internal building spaces.

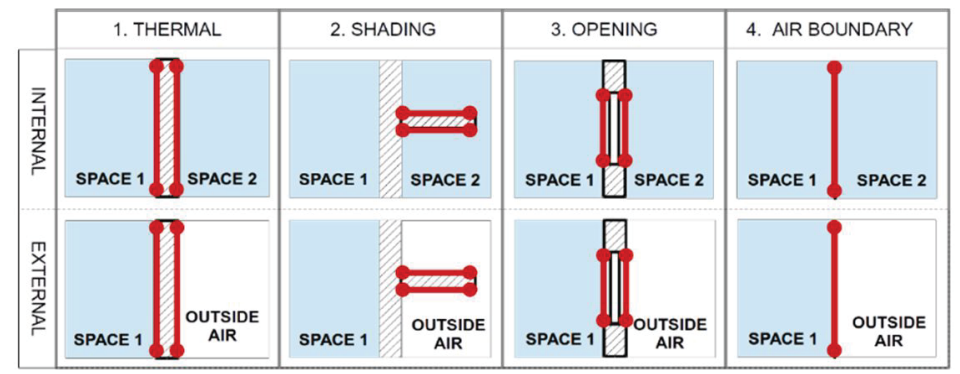

Figure 2: Boundary surface topology element types.

Although all of the above element types either external or internal are useful for the simulation model generation, only the elements belonging to categories 1, 3 and 4 are used directly as second-level space boundaries. 


\section{IFC FILE ENRICHMENT}

The geometric data of the surfaces of the boundary surface topology elements of type 1,3 and 4 of the building and their connectivity information are used in order to populate the IfcRelSpaceBoundary2ndLevel classes and enrich the final IFC file exported by the platform. An example of such enrichment is presented in Figure 3.

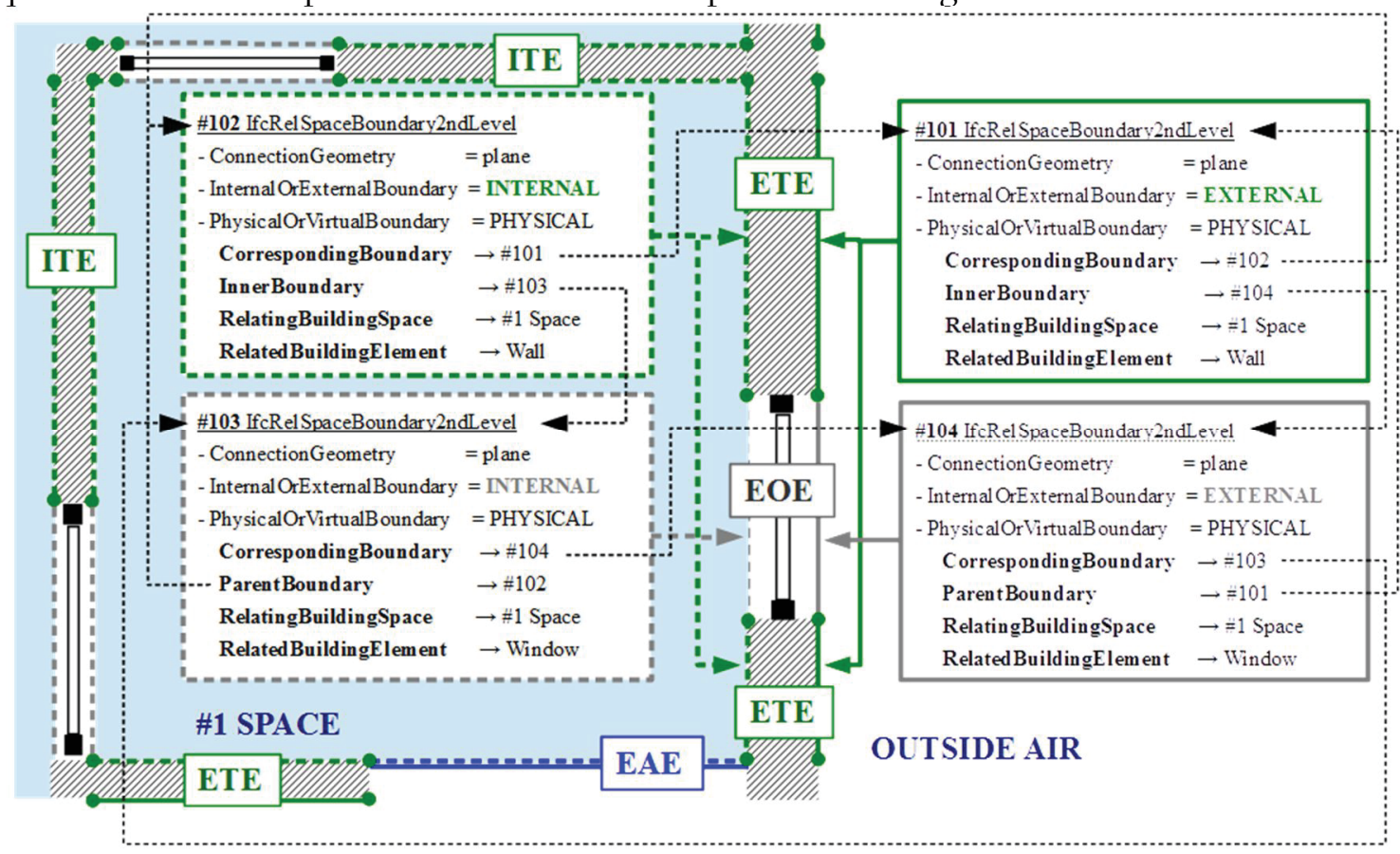

Figure 3: IFC classes updated by space boundary elements and BST elements.

As displayed in Figure 3, every IfcRelSpaceBoundary2ndLevel class is linked with other similar classes via three relation types which are:

- Parent - Inner. The parent space boundary surface contains multiple inner space boundary surfaces, as a wall contains multiple openings (\#101 contains \#104 in example of Figure 3).

- Correspondence. Every space boundary surface of type 2A (Bazjanac, V, 2010) has its opposite space boundary surface as its corresponding boundary surface (The corresponding surface of \#101 in the example of Figure 3 is surface \#102 and vice versa). Only second level space boundaries of type 2B (Bazjanac, V, 2010) and external shading surfaces, identified by CBIP algorithm, do not have corresponding boundary surfaces.

- Internal - External. Additionally, all boundary surfaces used in order to populate the IFC classes are characterized as INTERNAL if they are attached to an inner building space which is referred by the RelatingBuildingSpace field or EXTERNAL if they are attached to the outside air or ground.

- Physical - Virtual. If the boundary surface topology element of the space boundary surface is either, a thermal or an opening element then the space boundary is characterized as a PHYSICAL boundary. If the boundary surface topology element of the space boundary surface is an air boundary element, then the boundary surface is characterized as VIRTUAL boundary. 
The boundary surface topology generation involves the calculation of internal and external shading surfaces (boundary surface topology elements of type 2), out of which the internal shading surfaces can be characterized as second order space boundaries of type B (Bazjanac, V, 2010). These surfaces together with all the other type 2B space boundaries (which are also identified by the CBIP process) do not have corresponding boundary surfaces and are not used currently in the final IFC enrichment process.

\section{PlatForm ARCHITECTURE}

The need for multiple second-level space boundary topology generations might arise in two scenarios. In the first scenario, multiple clients, working on different projects require the second level space boundary topology to be generated for building energy performance simulation model generation purposes. In the second scenario, a single client requires generation of the second level space boundary topology for multiple buildings in a district environment.

For all these cases, parallel executions of the CBIP algorithm and of the BIM transformation module, by using virtual computing resources which are provided by cloud service providers, are implemented in the current platform. The distributed nature of the platform requires orchestration techniques and an Enterprise Service Bus (ESB) that connects the modules by using the asynchronous communication pattern. The functionality of the platform is obtained using a secure containerized environment hosted on dedicated servers. This containerized environment is a lightweight virtualization system that does not require virtual machines on physical hardware. By using docker containers the services are portable and distributed in a standardized manner providing flexibility and horizontal scalability. Within the platform three docker images have created and deployed to the cluster (see Figure 4), which are:

- CBIP Input XML Generation. This container consumes messages coming through the ESB which contain IFC data and produces the input xml data of the CBIP algorithm.

- CBIP Algorithm. This container consumes the generated input xml data and executes the CBIP algorithm, using the containerized version of Matlab Runtime. After the execution produces the output xml which contains the second level space boundaries.

- Enhanced BIM Generation. This container consumes messages which contain the Boundary Surface Topology data as well as the IFC data and produces the enhanced IFC data. Some input IFC data files have missing inverse references to identifiers due to exporter's bugs and as a result standard IFC viewers are not able to display them correctly. This container guarantees that the final enriched IFC file will be free of any of such issues.

The deployment of these images to the cluster requires operating systems especially designed for providing robust infrastructure into clustered environments. For this purpose the CoreOS was selected because provides only the minimal functionality required for deploying containers, together with built-in mechanisms for service discovery, configuration sharing and automated atomic updates.

Only a small fraction of the data contained in the IFC files are required for the operation of the web-based platform. These data refer to the geometrical shape 
representations of the buildings' architectural elements. Therefore, as an initial step this fraction of the data are extracted from the input IFC files and transformed into a CBIP compatible xml format. Similarly, CBIP services' output, is exported in an CBIP compatible $\mathrm{xml}$ format, before being used in order to enrich the initial IFC file in the output.

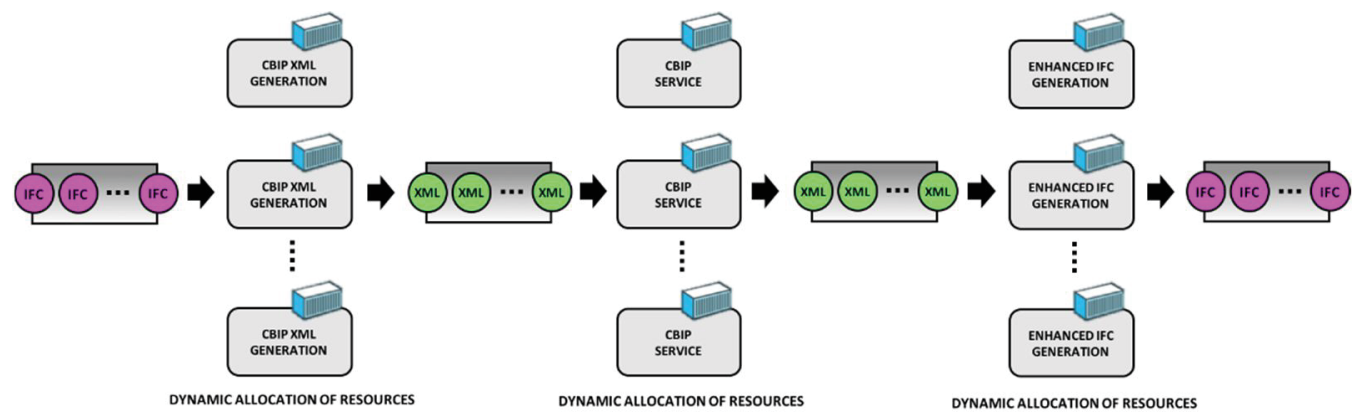

Figure 4: Orchestration of the docker containers using queues provided from the ESB.

The Graphical User Interface acts as producer and consumer to the platform simultaneously because on one side feeds the containers through the ESB with IFC data, on the other receives asynchronously the enhanced IFC data. Additionally, each container informs in real-time the users through a progress bar about the health of the running processes, by using the ESB broker and HTML5 web-sockets (see screenshot of Figure 5).

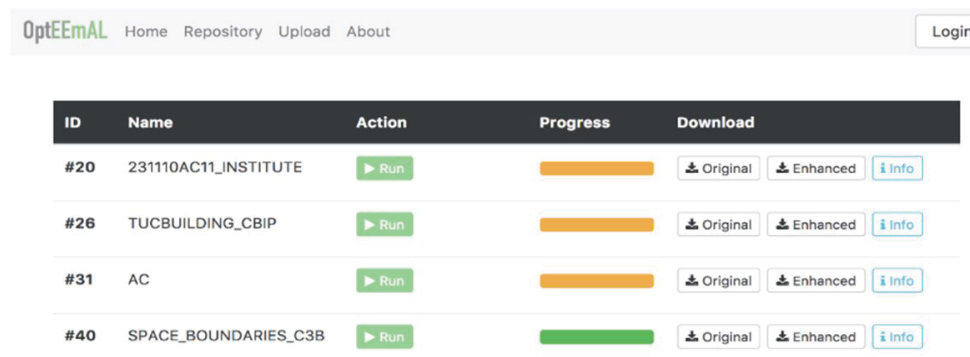

Figure 5: Screenshot of the platforms' graphical user interface.

The colours of the progress bar have the following meaning:

- Green. The process completed successfully.

- Orange. The process completed successfully but the initial IFC file contains one or more errors including design errors, orientation errors, documented when the info button is pressed.

- Red. The process failed due to severe error such as incompatible IFC version, corrupted files, bugs etc.

Multiple IFC files from various buildings in the same district can be processed at the same time, however the interactions among architectural elements, belonging to different IFC files, are not taken into account. Consequently, combination of multiple IFC files from the same district as a single input to the platform, is not supported at present, as it involves inter-building (inter-IFC) consistency checks. 


\section{EXAMPLE}

The use of the platform is demonstrated on a ten-story building in Cuatro de Marzo district in Valladolid in Spain which is displayed in part A of Figure 6. All the space boundaries of the building were identified correctly, as displayed for the fifth floor in parts B, C and D of Figure 6.

A. Demonstration building

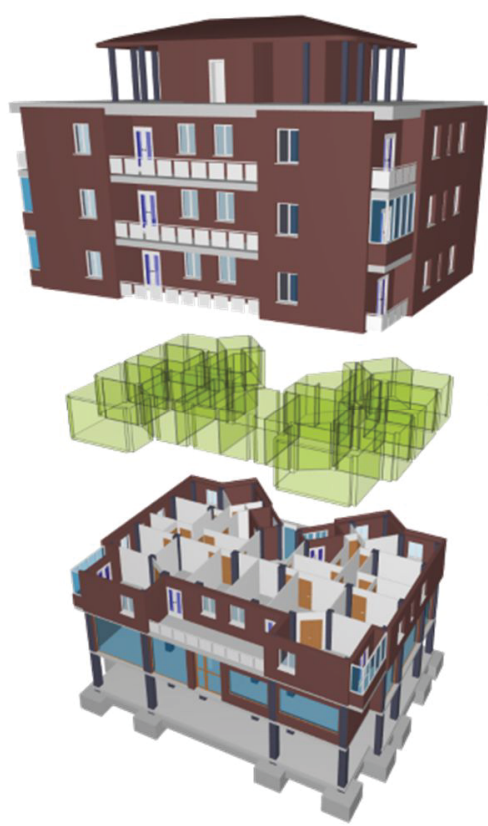

B. External / Internal wall space boundaries

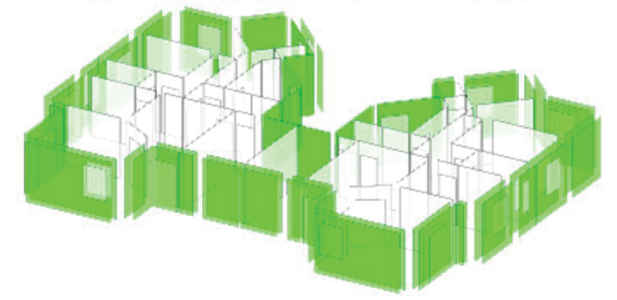

C. Internal slab space boundaries

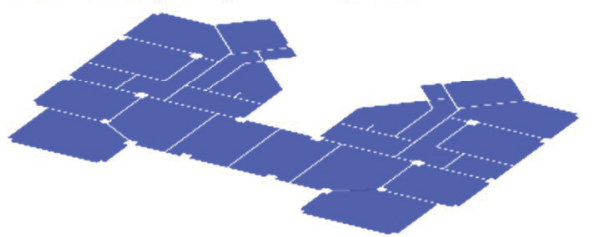

D. External / Internal opening space boundaries

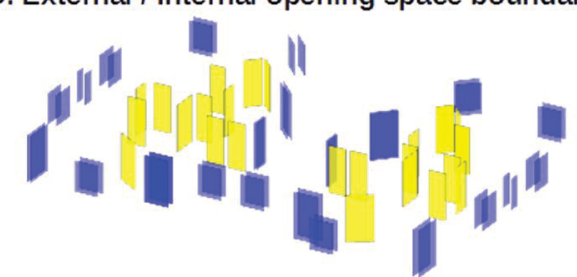

Figure 6: (A) Demonstration building located in Cuatro De Marzo, Valladolid, Spain, (B), (C) and (D) Space boundary topology obtained by the platform.

More specifically, in each building floor, five space boundary types were identified: external and internal wall space boundaries displayed with green and white colors respectively in part B of Figure 6, internal slab boundaries pictured with blue color in part $\mathrm{C}$ in Figure 6 and External and internal opening space boundaries displayed with blue and yellow colors, respectively in part D of Figure 6.

\section{COMPARISON WITH OTHER TOOLS}

The Space Boundary Tool (SBT) (Berkeley, n.d.), also performs space boundary topology calculations from IFC input data files. It also supports geometry simplification a function useful for BEP generation, which is not supported by the CBIP tool. However, SBT does not perform automatic generation of: virtual space partitions (Air boundaries), external shading surfaces and space boundaries originated from curtain wall elements (frames, plates, doors and windows) and is not IFC4 compatible, capabilities which are supported by the CBIP tool.

\section{CONCLUSIONS}

A cloud-based platform which can be used for the generation of the boundary surface topology of buildings (second level space boundaries and external shading surfaces), from 
IFC input data files was introduced. The platform uses distributed parallel computing resources in order to be able to accommodate heavy computation loads arising from multiple building designs, in a district environment. The platform's operation can be divided into two stages. The first is an error checking stage, where possible geometric errors affecting the quality of the generated second-level space boundary topology are detected and communicated back to the user using appropriate messages. The second-level space boundary topology is generated in the second stage by the Common Boundary Intersection Projection algorithm. The platform is validated on a demo building and the second-level space boundary content of the enriched IFC output file, is verified

Parts of the present platform, will be used in the European research project OptEEmAL for the automatic generation of multiple building simulation models, in a district environment, for retrofitting purposes.

\section{ACKNOWLEDGEMENTS}

Part of the work presented in this paper is based on research conducted within the project "Optimised Energy Efficient Design Platform for Refurbishment at District Level", which has received funding from the European Union Horizon 2020 Framework Programme (H2020/2014-2020) under grant agreement number 680676.

\section{REFERENCES}

Bazjanac, V. (2010). Space boundary requirements for modeling of building geometry for energy and other performance simulation. 27th CIB W78. Cairo.

Bazjanac, V. (2008). IFC BIM-based methodology for semi-automated building energy performance simulation. Lawrence Berkeley National Laboratory.

Bazjanac, V. (2009). Implementation of semi-automated energy performance simulation: building geometry. CIB W, (p. vol. 78). Istanbul.

Berkeley, L. (n.d.). Space Boundary Tool. Retrieved from http://simulationresearch.lbl.gov/projects/space-boundary-tool

Hirsch, J. (2014). DOE 2.1 . Retrieved from http://www.doe2.com

Jokela, M., Keinanen, A., Lahtela, H., Lassila, K. and Granlund, O. (1997). Integrated building simulation tool RIUSKA. IBPSA Building simulation conference. Prague, Czech Republic.

Karola, A., Lahtela, H., Hanninen, R., Hitchcock, R., Chen, Q., Dajka, S. and Hagstrom, K. (2002). BSPro COM-server interoperability between software tools using industrial foundation classes . Energy and Buildings, 34 (9), 901-907.

Lilis, G. N., Giannakis, G. I., and Rovas D. (2015). Detection and semi-automatic correction of gemetric inaccuracies in IFC files. IBPSA Building simulation conference. Hyderabad, India.

Lilis, G. N., Giannakis, G.I., and Rovas, D. (2017). Automatic generation of second-level space boundary topology from IFC geoemetry inputs. Automation in Construction, 76, 108-124.

O'Donnell, J. T. , Maile T. , Rose C., Mrazovic N., Morrissey E., Regnier C., Parrish C., Bazjanac V. (2014). Transforming BIM to BEM: Generation of building geometry for the NASA Ames sustainability base BIM. Lawrence Berkeley National Laboratory. 